Volume 8. No. 2, February 2020

International Journal of Emerging Trends in Engineering Research

Available Online at http://www.warse.org/IJETER/static/pdf/file/ijeter24822020.pdf

https://doi.org/10.30534/ijeter/2020/24822020

\title{
A YOLOv3 Inference Approach for Student Attendance Face Recognition System
}

\author{
Alvin Sarraga Alon ${ }^{1}$, Cherry D. Casuat ${ }^{2}$, Mon Arjay F. Malbog ${ }^{3}$, Rufo I. Marasigan Jr. ${ }^{4}$, Joshua S. \\ Gulmatico ${ }^{5}$ \\ ${ }^{1}$ Technological Institute of the Philippines, Manila Philippines, aalon.cpe@tip.edu.ph \\ ${ }^{2}$ Technological Institute of the Philippines, Manila Philippines, ccasuat.cpe@tip.edu.ph \\ ${ }^{3}$ Technological Institute of the Philippines, Manila Philippines, malbog.monarjay@gmail.com \\ ${ }^{4}$ Technological Institute of the Philippines, Manila Philippines, rufo.marasigan.jr@ @ip.edu.ph \\ ${ }^{4}$ Technological Institute of the Philippines, Manila Philippines, jgulmatico.cs@tip.edu.ph
}

\begin{abstract}
Checking attendance in a classroom is a factor contributing to the final performance of the students in the course. For both students and professors, attendance checking by name is very time-consuming and, in particular, the latter is very susceptible to simple attendance fraud. The study used a Face Recognition based attendance method using the YOLOv3 approach as an alternative. The system, based on face-detection and face-recognition algorithms, automatically recognizes, and marks attendance by recognizing the student. The experimental result shows that by using the trained model with a training accuracy of $98.01 \%$, the proposed attendance system achieved $94 \%$ face recognition efficiency.
\end{abstract}

Key words: attendance, deep learning, face recognition, yolov3

\section{INTRODUCTION}

A typical approach to record the attendance of the students are carried out either by reciting the name or thru the signing method on an attendance sheet that passes amongst students at the beginning of the class [1]. However, these approaches have various drawbacks. First, it proves inefficient in terms of overhead leading to wastage of time. Second, it can potentially fail to support the genuine counting of students that may lead to attendance fraud particularly in a large class, where a certain student might just mark on the attendance sheet for other students who are not physically present in the session. To mitigate or prevent such drawbacks, sometimes the professor or instructor manually calls out one by one those students in order to guarantee the authenticity of the attendance sheet [2]. These approaches have a negative influence on the efficiency and time of the lecture.
In recent trends in modern industries, automated attendance system has been anticipated by using several identification strategies like biometric recognitions such as palm vein [3], [4], fingerprint [5], [6], and face recognition [7], [9] to recognized and mark the students as present. The other proposed systems for tracking attendance used RFID [10], [11], barcode [12], QR code [13], mobile devices that uses Near Field Communication (NFC) [14], and some systems developed in a portable device like smartphone [15].

The study proposed a class attendance scheme that employs face-recognition approach. The system proposed a much more reliable attendance monitoring which can reduce drawbacks such as the wastage of time and fraud attendance. Due to the accuracy of the system, for the best interest of the students, absences were quickly identified resulting in a faster and appropriate action that can be used by the faculty or school administrator. The system was done by extracting and recognizing the features of the face in order to determine the correct face identity from an individual and getting the attendance marked by validating it with the provided datasets. The system used a YOLOv3 approach for an extremely fast and accurate face-recognition attendance system. This deep learning algorithm was used to compare a live video or image capture to verify the individual's identity for attendance markings.

\section{RELATED LITERATURE}

In the study of [3], the biometric palm vein is becoming the most encouraging physiological trademark for creating an effective identification framework. The paper addresses the new measurement in order to create biometric trait key, instead of just template free key generation derived by methods of comprehensive information security and pattern recognition. The study gave a new and profound calculation for the subject being recognized. The testing gives a result with a $99.47 \%$ accuracy rate for the recognition of the subject. 
Another study related to palm's vein conducted by [4] that recognition of palm vein patterns is one of the newest studies using today's biometric techniques. The paper describes a palm vein identification system that makes use of blood vessel patterns as a specific identifying factor. Knowledge of the veins is hard to replicate, as the veins are unique to the human body. The design specifics of the palm vein collection system are examined, and the paper also investigated the functionality of preprocessing and palm vein image extraction. The proposed capture method creates a tiny archive of palm-vein images. Experimental results indicated that an acceptable level of performance at the small palm vein database is reached by the designed system.

The study of [5] also recommended an attendance system that uses an automated fingerprint biometric to reduce the issues with traditional and manual attendance checks. The goal of the study is to create an online attendance system which integrates GPS fingerprint modules. The fingerprint module ZFM-20 is used as the system's key input, as well as a control tool as an entrance to access the entire system. The study uses the GPS module to calculate the user's location and then send it to the smartphone. Arduino device was used in the system which automatically sends SMS regarding the user's location data to the parties concerned. Another study conducted by paper [6] implemented the design technique for a simple and high real-time Zigbee-biometric system for simple management and time-saving attendance using fingerprints from employees at any organization along with inbound and outbound log maintenance for employees. The system worked first by scanning the fingerprints of the employee and an identification number is assigned as their registration. The machine compared the current fingerprint trends and the interaction from various points in the fingerprint to the identification log during the attendance period as workers press their fingerprints against the scanner.

A classroom attendance screening system based on face recognition was suggested in the study of [7], [8]. The paper's aim is to implement an automated, face-recognition smartphone attendance management system that does not require any additional devices. To this end a Euclidean distance-based facial recognition filtering system was developed, measured using three facial recognition techniques, namely Fisherfaces, Local Binary Pattern, and Eigenfaces. The research also includes three different mobile applications that enable parents, students, and teachers to control and conduct the attendance process in real time on their smartphones. A similar study carried out by [9] for face recognition, the system was developed by combining universal components to construct a portable device to monitor student attendance.
The study of [10] discusses the creation of an on-campus attendance simulation utilizing RFID connected to the database and attendance records. The automatic program simplifies the on-campus attendance phase and allows it more organized. The simulation used an RFID tag to identify the id card of the students. The user is linked to the attendance data system so that all attendance details can be transferred automatically to the archive and used for data management later. The attendance obtained by placing the tag next to the person and the specific information will be immediately passed to the database and shown on the attendance app. Another RFID-based attendance system by [11] combines the RFID and IoT (Internet of Things). For better performance, the study used the Cloud as storage.

The study made by [12] used an SMS-based attendance system. Application-based student participation SMS Gateway was made to ensure students attend classes through the following steps: first, students will send the teacher the ID card on duty, then the instructor scans the barcode printed on the document, and then, the data will be registered and immediately stored in the database. The details will be periodically collected on a daily basis, and then it will be sent to their parents who have their mobile devices recorded on the same date and time to receive information regarding their children attending class.

Another method of attendance was worked out through the study of [13], the paper suggested a smartphone-based attendance program. The mobile apps allow simple internet connectivity and use Quick Response (QR) software-based methodology as a method for directly reporting field staff connecting to the web portal with the respective recipient company attendance. The paper discusses the details for applying the proposed system at high level.

In the study of [14], mobile software was developed which allows the reporting of the signature system applied in academic institutions as a computerized system with a minimum failure rate. NFC tags can be used to effectively monitor their participation according to their position and contact period due to the smart attendance system developed utilizing NFC technology.

Many students have some sort of smart device and these can be used to create an app and allow students to sign in to participate. The work of [15] using its established Muffin software has created one possible solution. Muffin is a mobile application that is available to students on their personal mobile devices, a Bluetooth modulated Arduino Uno board, and a computer app. 


\section{MATERIALS AND METHOD}

\subsection{Block Diagram}

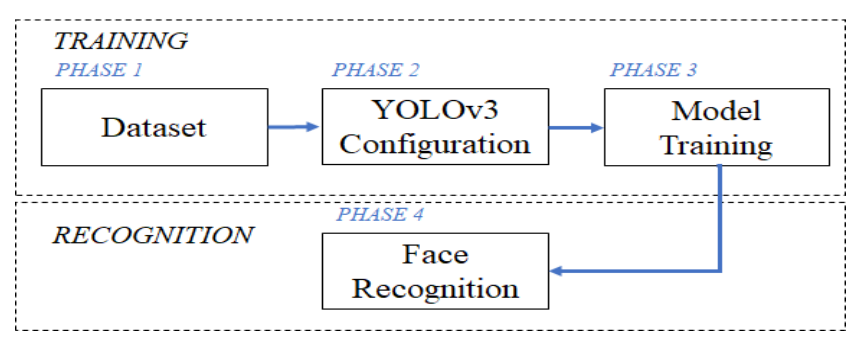

Figure 1: Architecture of Student Attendance Face Recognition

Figure 1 shows the block diagram of the system. The upper portion shows the training process while the lower portion is for recognition. Each part will be discussed in the succeeding section.

\subsection{Phase 1 - Dataset}

Table 1: Dataset and Materials

\begin{tabular}{ll}
\hline Materials / Components & Description \\
\hline Software & Ubuntu 16.04 \\
& Python 3.6 \\
& OpenCV \\
& CUDA 9.0 \\
& CUDNN 9.0 \\
& Anaconda \\
& Darknet \\
& Intel Core i7 2.8 GHz \\
Hardware & 4.0 GB RAM \\
& NVIDIA GeForce GTX1050 \\
Data & Data that is gathered will be captured by a camera.
\end{tabular}

Table 1 shows all the software and dependencies used in the study. A web camera was used to produce a series of images for dataset generations. The type of data is in the format of an image. The data that was acquired is a collection of images that contains different angles of the faces of each student. The data should be clear enough to be used for training.

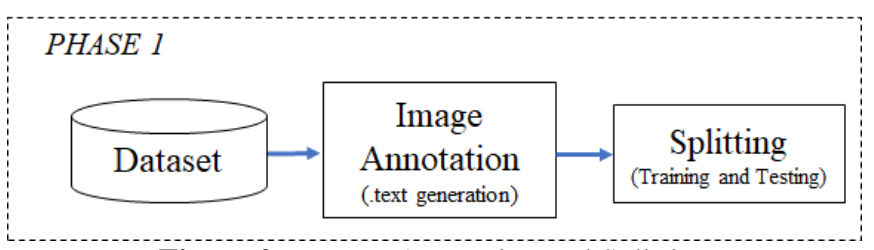

Figure 2: Dataset Annotation and Splitting

Phase one is about dataset preparation that includes annotation and splitting as shown in Figure 2. The dataset must be annotated in the format required by the Convolution Neural Network (CNN) trainer. For the YOLOv3, a ".txt" file is needed for each image file in the same directory with the same name. Each file will contain the object class, $x, y$, width, and height. The object class is an integer value from zero up to classes minus one. The $\mathrm{x}, \mathrm{y}$, width, and height are float values that contain the relative width and height of the image. Then, the dataset generated will be separated from the test data and training data.

\subsection{Phase 2 - YOLOv3 Configuration}

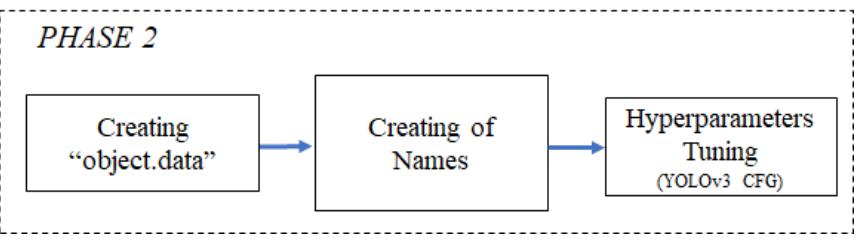

Figure 3: YOLOv3 Configuration

For the second phase as shown in Figure 3, the YOLOv3 configuration files are then prepared. Create first a ".data file", This will contain the number of classes, the addresses to your list of train data, test data, obj.names, and location of the backup. Also, the ".cfg file", the filter, and the class value should be edited accordingly.

\subsection{Phase 3 - Model Training}

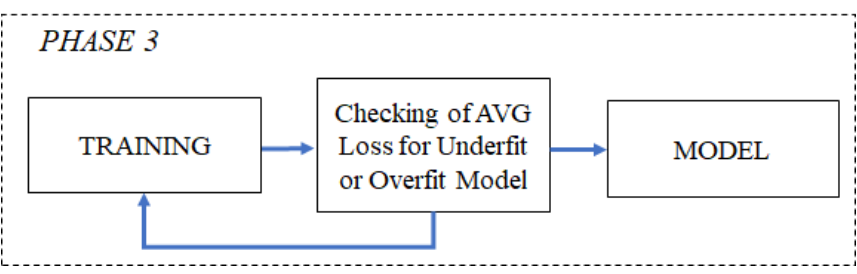

Figure 4: Model Training

The third phase is all about the model training as shown in Figure 4 . The training used a GPU-based computing machine to decrease the training time. After the training, the model was created. The value of the average loss has been observed to determine if the model is underfitting or overfitting. The final model was then used for recognizing the faces from the camera for inferencing.

\subsection{Phase 4 - Face Recognition}

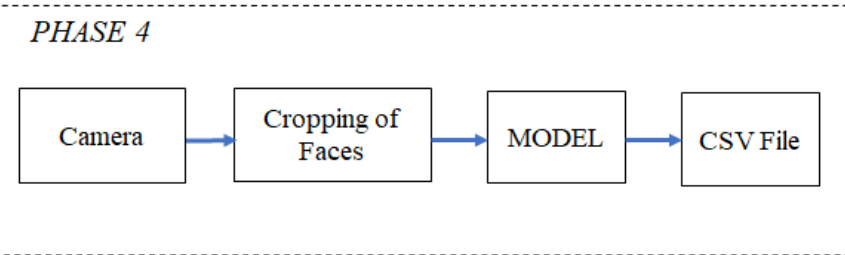

Figure 5: Face Recognition

Figure 5 shows the fourth and last phase, model inferencing. The camera will serve as an input device. The model that was generated from the third phase will be applied to each frame from the capturing device. It will now detect all the faces that the model has seen in each of the frames. 


\section{RESULTS AND DISCUSSIONS}

\subsection{Phase 1 - Dataset}

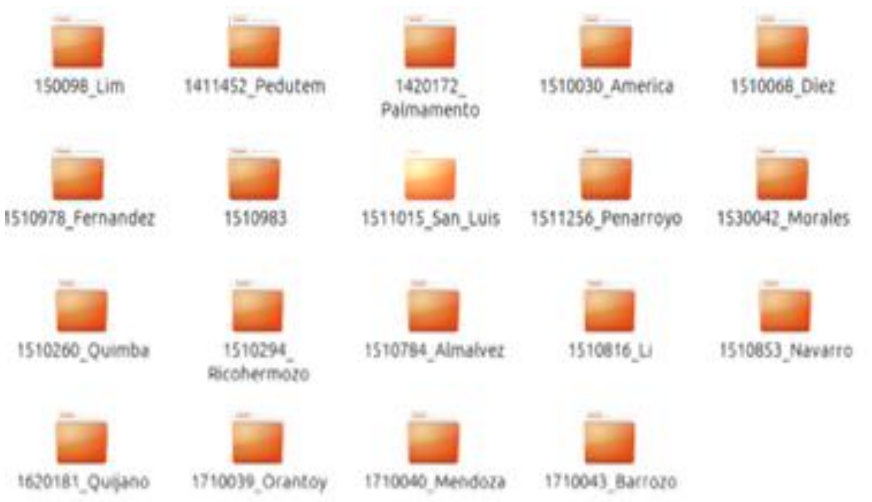

Figure 6: Folder of Datasets

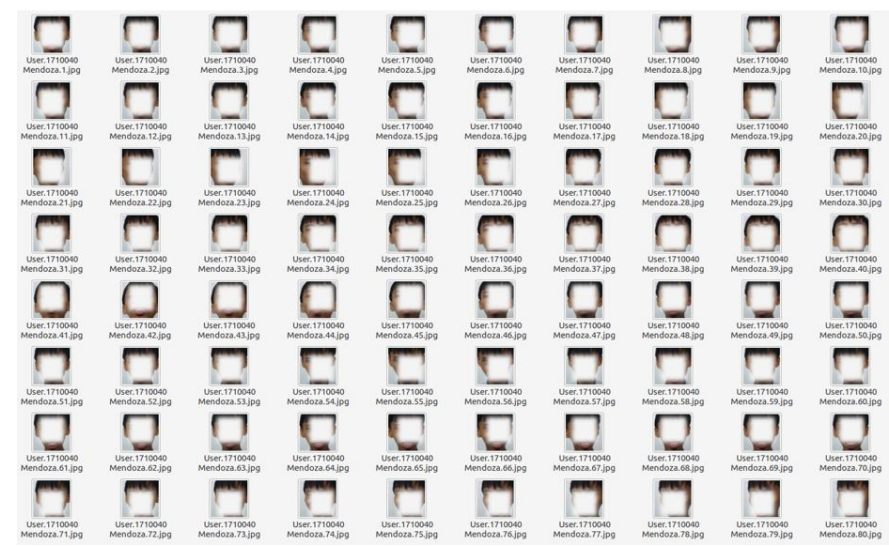

Figure 7: Collection of Images

Figure 6 shows the dataset that was used for face recognition. Each folder contains 500 JPEG format images. Each image includes the faces of each student in the classroom. Figure 7 shows an example of what does the folder in Figure 6 contains. Unfortunately, there was noise included in the dataset, some of the photos taken was really not a face. Those pictures were manually handpicked and deleted to have a clean dataset. Therefore, some of the folders contain less than 500 images.

All of these images must be manually annotated with labels. Each row entry in a label file represents a single bounding box in the image. The splitting of the dataset between training and testing data has been done. Figure 8 shows the split training and testing image data. The computed values from annotations were manually checked with a calculator to ensure annotation correctness. Also, it was compared to an existing object detection to prove that the text files produced were correct.

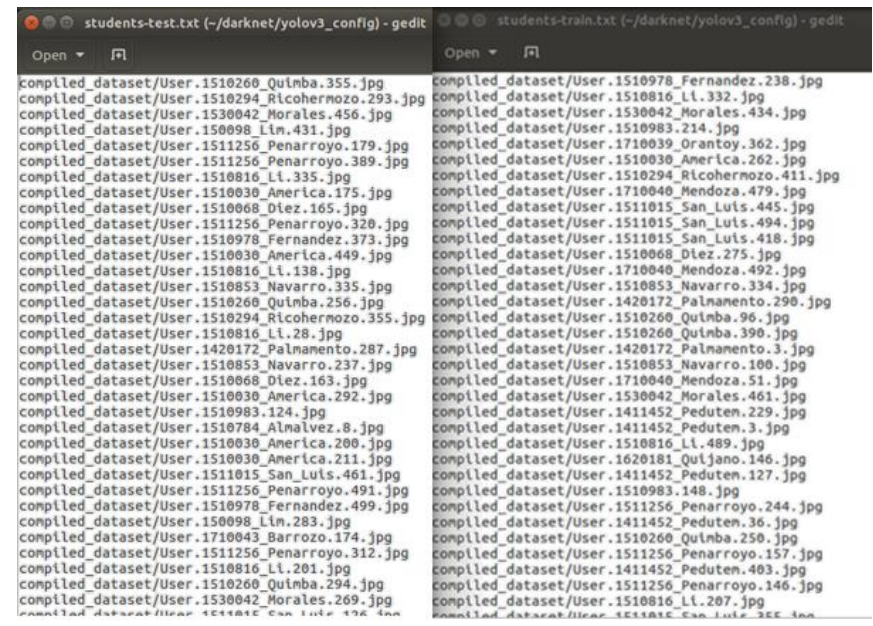

Figure 8: Training and Testing Datasets

\subsection{Phase 2 - YOLOv3 Configuration}

For the second phase, Darknet has been prepared. Installation for Windows is different. For Linux, AlexeyAB's Darknet is cloned to the system. The "makefile" has been edited by changing GPU, CUDA, and OPENCV to 1 . This has been done to increase the performance of the training. The YOLOv3 configuration files were then prepared. The ".data file" was created. This contains the number of classes, the address to your list of train data, test data, obj.names, and location of the backup. The backup folder contains the weights generated by the Darknet. For the study, the file name is named as "students.data" as shown in Figure 9.

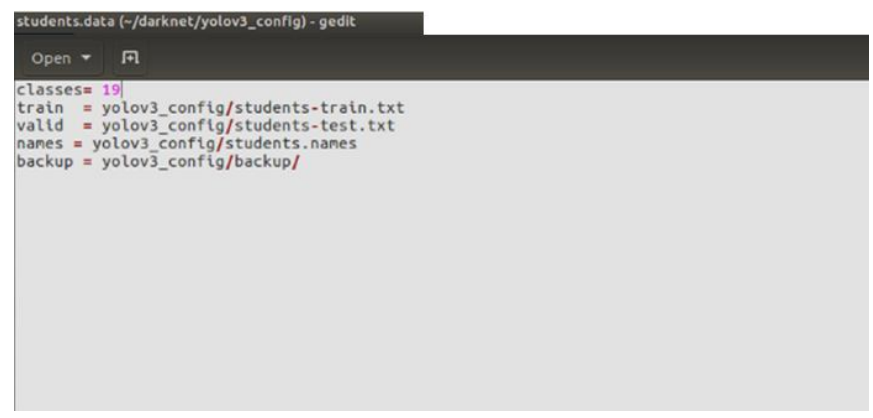

Figure 9: students.data Information

Table 2: YOLOv3 Configuration File Edit

\begin{tabular}{ll}
\hline Line & Description \\
\hline 3 & set batch $=64$, that means we'll use 24 images for each training phase \\
4 & $\begin{array}{l}\text { set subdivisions }=64 \text {, dividing a batch by } 8 \text { to reduce GPU VRAM } \\
\text { requirements. } \\
\text { set filters }=(\text { classes }+5) * 3 \text { in our case filters }=72\end{array}$ \\
610 & set classes $=19$, the number of categories we want to detect \\
689 & set filters $=($ classes +5$) * 3$ in our case filters $=72$ \\
696 & set classes $=19$, the number of categories we want to detect \\
776 & set filters $=($ classes +5$) * 3$ in our case filters $=72$ \\
783 & set classes $=19$, the number of categories we want to detect
\end{tabular}


The "yolov3.cfg file" has been edited as shown in Table 2 . The filters and class value has been changed. The study just duplicated the yolov3.cfg file, and made the following edits. The study used the pre-trained convolutional weights of "darknet53.conv.74".

\subsection{Phase 3 - Model Training}

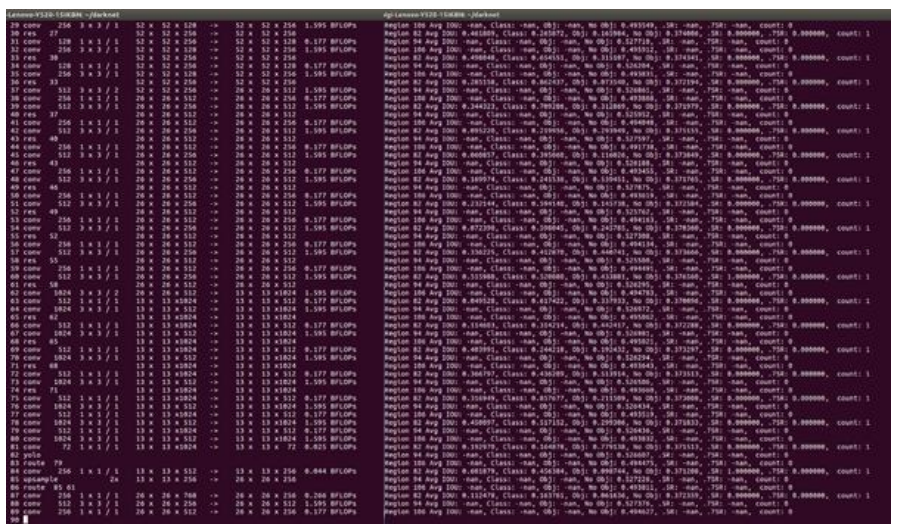

Figure 10: Training Process

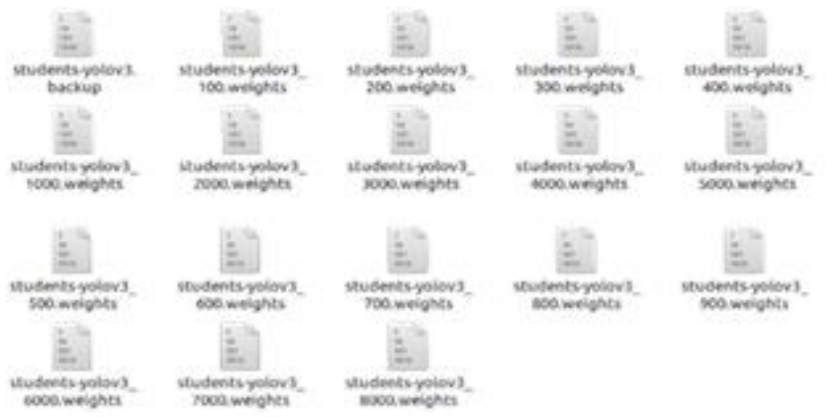

Figure 11: Weights Generated

Figure 10 shows the model training, the process took for more than 2 days straight. The study has edited the darknet codes to change the checkpoints, weights only save every 1000 iterations as shown in Figure 11.

\subsection{Model Accuracy}

AP (Average accuracy) is a metric for measuring object detector accuracy, such as SSD, Faster R-CNN, etc. It is the average precision that has accounted for different recall values. For this section, the study used mAP for evaluating the model accuracy. The mAP was generated from each 1000th weight checkpoint that the Darknet produced as shown in Figure 12.

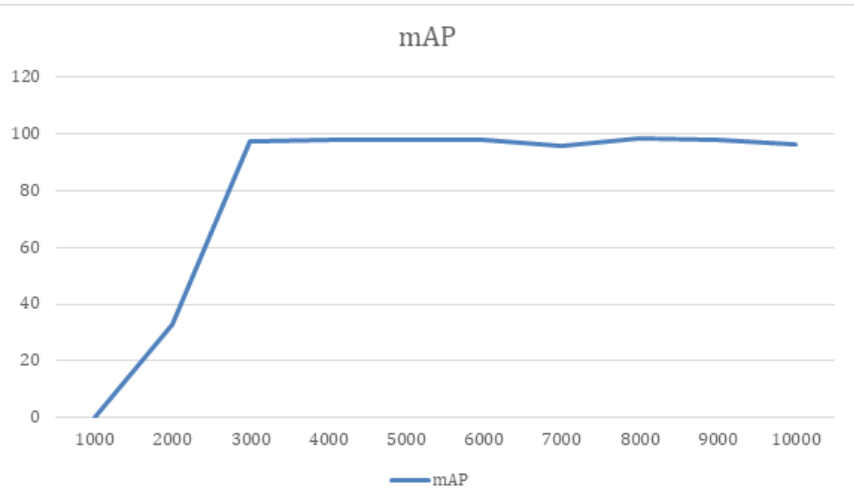

Figure 12: $\mathrm{mAP}$ for each $1000^{\text {th }}$ Weight

Based on the mAP data from Figure 12, the study used the 4000th weight, since it has produced the highest mAP value of $98.01 \%$.

\subsection{Phase 4 - Face Recognition}

Figure 13 shows the simple user interface (UI) of the attendance face recognition. A CSV file will be generated when the student recognized by the camera.

\begin{tabular}{|c|c|c|c|c|}
\hline \multirow{2}{*}{$-\square$} & \multirow{2}{*}{ 口 } & \multirow{2}{*}{$\mathbf{x}$} & DATE_TIME & NAME \\
\hline & & & 2019_03_26_11_48_41 & 1510098_Lim \\
\hline & & & 2019_03_26_11_48_42 & 1510098_Lim \\
\hline \multirow{2}{*}{\multicolumn{3}{|c|}{ Start }} & 2019_03_26_11_48_42 & 1510098_Lim \\
\hline & & & 2019_03_26_11_48_48 & 1510098_Lim \\
\hline \multicolumn{3}{|c|}{ Generate } & 2019_03_26_11_49_07 & 1510978_Fernandez \\
\hline & & & 2019_03_26_11_49_08 & 1510978_Fernandez \\
\hline
\end{tabular}

Figure 13: Simple UI and CSV Data

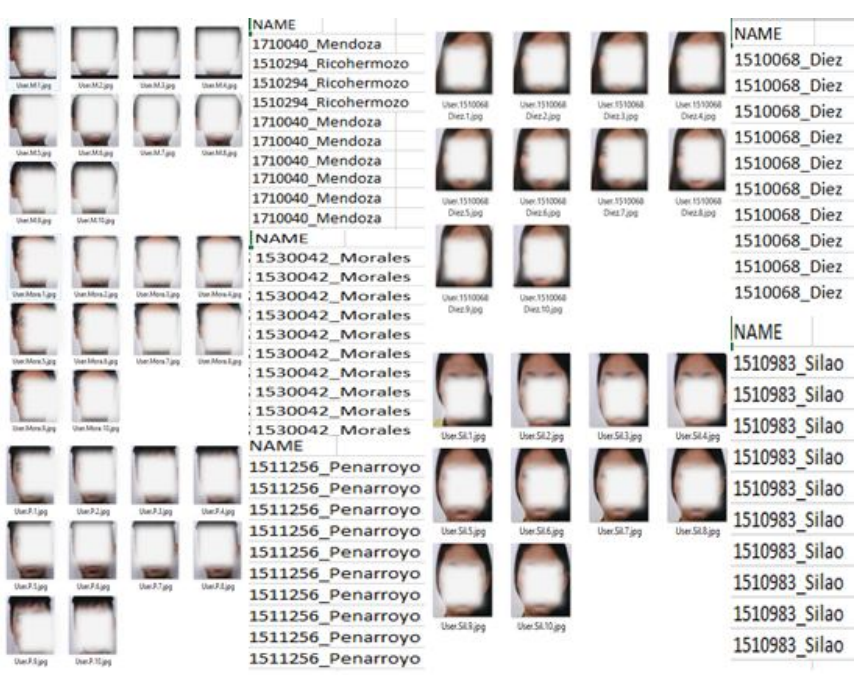

Figure 14: Student Attendance Face Recognition Testing

During the inference of the model, actual testing has been done. Five students have been used for testing as shown in Figure 14, 10 frames were used from each student. As shown in Table 3, for the student Mendoza, 7 out of 10 frames were correctly recognized. The remaining students were correctly recognized by the inference and gathered a $100 \%$ accuracy. 
Alvin Sarraga Alon et al., International Journal of Emerging Trends in Engineering Research, 8(2), February 2020, 384 - 390

The overall experimental accuracy of the system is $94 \%$.

Table 3: Summary of Testing

\begin{tabular}{lll}
\hline Student Names & \# of Frames Correctly Recognized & Accuracy \\
\hline Diez & $10 / 10$ & $100 \%$ \\
Mendoza & $7 / 10$ & $70 \%$ \\
Morales & $10 / 10$ & $100 \%$ \\
Pennaroyo & $10 / 10$ & $100 \%$ \\
Silao & $10 / 10$ & $100 \%$ \\
\hline Total & & $94 \%$
\end{tabular}

\section{CONCLUSION}

The study presented a class attendance program based on YOLOv3 deep learning architecture that used face-recognition. The system requested that each listed student capture his / her face-image before a web camera. The facial recognition model then recognized the inferential image automatically. The experimental testing shows that the study achieved a $94 \%$ face recognition performance by using the trained model with a $98.01 \%$ training accuracy. The study shows that you don't always have to rely on general-purpose trained models in situations in order to train the deep learning model where these models are overkill to your problem and performance issues involved. Don't overestimate the power of spending time designing the problem-specific custom neural network. Such unique networks will be a far better solution than the general ones. Training the models often takes too much time, depending on your computer's output.

\section{ACKNOWLEDGEMENT}

The authors wish to express their gratitude to the Technological Institute of the Philippines' MR-SUAVE High-Performance Computing Laboratory.

\section{REFERENCES}

1. M. M. Islam, M. K. Hasan, M. M. Billah and M. M. Uddin. Development of smartphone-based student attendance system, in 2017 IEEE Region 10 Humanitarian Technology Conference (R10-HTC), 2017, pp. 230-233. https://doi.org/10.1109/R10-HTC.2017.8288945

2. B. K. P. Mohamed, C. V. Raghu. Fingerprint attendance system for classroom needs, in 2012 Annual IEEE India Conference (INDICON), New York, 2012, pp. 433-438. https://doi.org/10.1109/INDCON.2012.6420657

3. S. D. Raut, V. T. Humbe and A. V. Mane. Development of biometric palm vein trait based person recognition system: Palm vein biometrics system, in Proc. 1st International Conference on Intelligent Systems and
Information Management (ICISIM), Aurangabad, 2017, pp. 18-21.

https://doi.org/10.1109/ICISIM.2017.8122140

4. H. Zhang and D. Hu. A Palm Vein Recognition System, in 2010 International Conference on Intelligent Computation Technology and Automation, Changsha, 2010, pp. 285-288. https://doi.org/10.1109/ICICTA.2010.425

5. L. Kamelia, E. A. D. Hamidi, W. Darmalaksana and A. Nugraha. Real-Time Online Attendance System Based on Fingerprint and GPS in the Smartphone, in 2018 4th International Conference on Wireless and Telematics (ICWT), Nusa Dua, 2018, pp. 1-4.

6. M. Kamaraju and P. A. Kumar. Wireless fingerprint attendance management system, in 2015 IEEE International Conference on Electrical, Computer and Communication Technologies (ICECCT), Coimbatore, 2015, pp. 1-6.

7. P. M.V.D, Human activity recognition using Deep Learning, International Journal of Emerging Trends in Engineering Research, vol. 7, no. 11, pp. 536-541, 2019. https://doi.org/10.30534/ijeter/2019/227112019

8. J. P, Facial expression detection of all emotions and face recognition system, International Journal of Emerging Trends in Engineering Research, vol. 7, no. 12, pp. 778-783, 2019.

https://doi.org/10.30534/ijeter/2019/087122019

9. T. T. Oo, and A. M. Africa. Emotion Basic action using Face Recognition and Detection ANN, International Journal of Emerging Trends in Engineering Research, vol. 7 No. 11, pp. 610-616, November 2019. https://doi.org/10.30534/ijeter/2019/337112019

10. D. Eridani and E. D. Widianto. Simulation of attendance application on campus based on RFID (radio frequency identification), in 2015 2nd International Conference on Information Technology, Computer, and Electrical Engineering (ICITACEE), Semarang, 2015, pp. 460-463. https://doi.org/10.1109/ICITACEE.2015.7437850

11. T. Sharma and S. L. Aarthy. An automatic attendance monitoring system using RFID and IOT using Cloud, in 2016 Online International Conference on Green Engineering and Technologies (IC-GET), Coimbatore, 2016, pp. 1-4.

https://doi.org/10.1109/GET.2016.7916851

12. M. Benny Chaniago and A. Junaidi. SMS Gateway and barcode technology for presence of students in SMK Unggulan Terpadu PGII Bandung: A case study, in 2016 4th International Conference on Cyber and IT Service Management, Bandung, 2016, pp. 1-4. https://doi.org/10.1109/CITSM.2016.7577576

13. K. Navin, A. Shanthini and M. B. M. Krishnan. A mobile based smart attendance system framework for tracking field personals using a novel $\mathbf{Q R}$ code based technique, in 2017 International Conference On Smart Technologies For Smart Nation (SmartTechCon), Bangalore, 2017, pp. 1540-1543. 
Alvin Sarraga Alon et al., International Journal of Emerging Trends in Engineering Research, 8(2), February 2020, 384 - 390

https://doi.org/10.1109/SmartTechCon.2017.8358623

14. M. Baykara, U. Gürtürk and E. Karakaya. NFC based smart mobile attendance system, in 2017 International Conference on Computer Science and Engineering (UBMK), Antalya, 2017, pp. 937-941.

https://doi.org/10.1109/UBMK.2017.8093571

15. S. M. Čisar, R. Pinter, V. Vojnić, V. Tumbas and P. Čisar. Smartphone application for tracking students' class attendance, in 2016 IEEE 14th International Symposium on Intelligent Systems and Informatics (SISY), Subotica, 2016, pp. 227-232.

https://doi.org/10.1109/SISY.2016.7601502 